\title{
SCALE INVARIANT OPTICAL CORRELATION USING MELLIN TRANSFORMS
}

\author{
David CASASENT and Demetri PSALTIS \\ Carnegie-Mellon University, Department of Electrical Engineering, \\ Pittsburgh, Pa. 15213, USA
}

Received 11 December 1975, revised version received 15 January 1976

\begin{abstract}
The use of optical Mellin transforms in matched spatial filter correlation of imagery that differs in scale is discussed. Theoretical considerations and initial experimental results are included. The intensity of the correlation peak is found to be independent of the input scale, and the scale difference between the two functions to be correlated can also be extracted from the location of the correlation peak.
\end{abstract}

\section{Introduction}

Optical pattern recognition is one of the most powerful operations possible on a coherent optical system. In the conventional optical correlator, one function is placed in the input plane and a Fourier transform hologram (FTH) or matched spatial filter [1] of the second function is positioned in the Fourier transform plane. The resultant light distribution in the output plane contains the correlation of the two functions [2]. This initial correlator has been used to demonstrate the feasibility of spatial pattern recognition in many applications [3]. However, the transition from these initial feasibility demonstrations to practical, competitive and reliable commercial systems requires that orientation and scale problems be overcome. The positioning and orientation requirements on the input and spatial filter are quite severe and positioning accuracies of a few $\mu \mathrm{m}$ are normally required.

Moreover, the scale of the function from which the FTH is formed must precisely match that of the input function or a large loss in the signal-to-noise ratio (SNR) of the correlation peak will occur. The effects of the percent scale difference $a$ between the input and matched FTH functions on the SNR of the correlation peak are shown in fig. 1 for an aerial image. The autocorrelation yielded a peak correlation SNR of nearly $30 \mathrm{~dB}$, while the SNR for a small $2 \%$ scale charge had decreased to $3 \mathrm{~dB}$. Several methods have been proposed to overcome this practical problem. One solution is

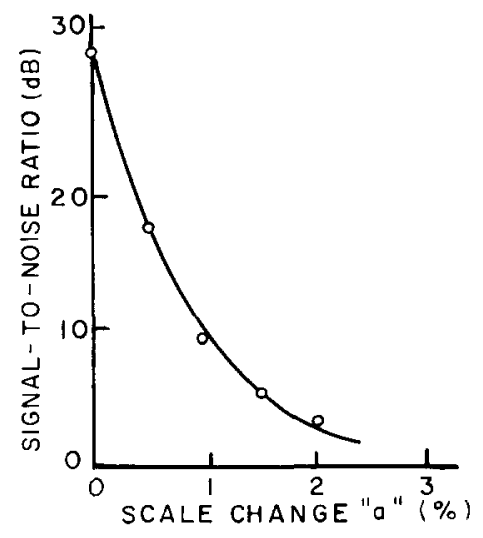

Fig. 1. Plot of SNR (signal-to-noise ratio) of the correlation peak vs. the percent scale change $a$ between the functions correlated.

the use of multiple holographic filters for different scaled versions of the input. This requires the accurate synthesis of multiple spatially or angularly coded matched filters on a holographic storage material. Besides practical synthesis problems, this approach suffers from a loss in diffraction efficiency proportional to the square of the number of multiplexed filters. In an alternate approach, the input is placed behind the transform lens rather than in front of it. As the distance from the input to the transform plane is varied, so is the scale of the transform. While this is a viable solution when scale differences of $20 \%$ or less 

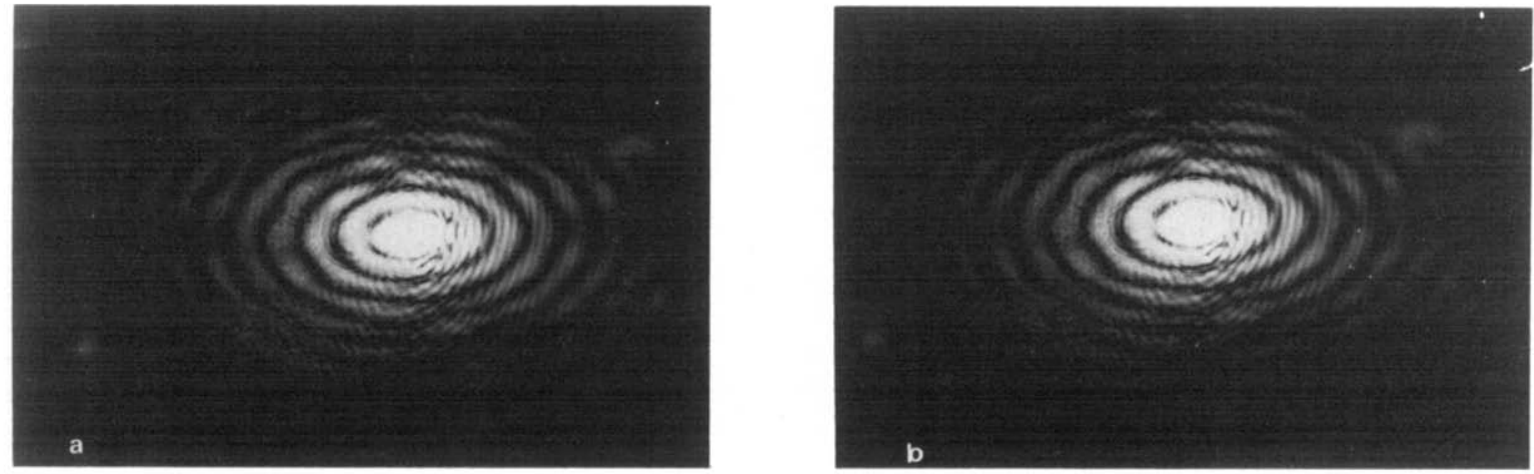

Fig. 2. Optical Mellin transform of two circular input functions of diameter $d$ (fig. a) and $2 \mathrm{~d}$ (fig. b).

are encountered, it requires the mechanical movement of components and thus does not retain the real-time processing potential of an optical system.

An attractive solution to this problem is the use of operations other than the Fourier transform. The Mellin transform is especially useful since it is scale invariant $[4,5]$ and has recently been implemented digitally [5] as well as optically [4]. In this paper, the previously reported optical synthesis of a Mellin transform [4] will be briefly reviewed, several methods of optical scale invariant correlation using this transform will be discussed, and initial experimental results of the correlation of objects which differ in scale by $200 \%$ with no loss of SNR will be presented.

\section{Optical Mellin transforms}

The Mellin transform [6] arises in the analysis of time-varying networks [7], in space-variant image restoration [8], and in the analysis of signals with multicomponents of the same form but different width [9]. Its use has also been suggested for speech processing [5]. The two-dimensional Mellin transform $M$ of a function $f$ along the imaginary axis is defined by

$M(\mathrm{j} u, \mathrm{j} v)=\int_{0}^{\infty} f(x, y) x^{-\mathrm{j} u-1} y^{-\mathrm{j} v-1} \mathrm{~d} x \mathrm{~d} y$.

For simplicity only the one-dimensional case will be considered. Our interest lies in the optical synthesis of this transform. Since the basic operation performed by a lens is the Fourier transform, the optical synthesis must involve this operation. Fortunately, it has been shown [4] that a simple variable change $x=\exp \xi$ on the input $f(x)$ followed by a Fourier transform of the scaled function $f(\exp \xi)$ will yield the Mellin transform of $f(x)$,

$M(\mathrm{j} \omega)=\int_{0}^{\infty} f(\exp \xi) \exp (-\mathrm{j} \omega \xi) \mathrm{d} \xi$

For simplicity $M(\mathrm{j} \omega)$ will be written as $M(\omega)$ hereafter.

Several methods of implementing this transform in real-time on optically and electronically addressed devices have been previously discussed. The optical Mellin transform of two simple circular input objects that differ in scale by a factor of $200 \%$ are shown in figs. $2 a$ and $2 b$. Both transforms were formed by the same lens system and no adjustments were made in photographing the outputs. As predicted, the two transforms are identical even though the inputs differ widely in scale.

\section{Scale invariant correlation}

The basis for conventional correlation using matched spatial filters is the translational or shift invariance of the Fourier transform. If $M_{1}(\omega)$ is the Mellin transform of a function $f_{1}(x)=f(x)$, then the Mellin transform $M_{2}(\omega)$ of a scaled version $f_{2}(x)=f(a x)$ of $f_{1}(x)$ can be shown from eq. (1) to be

$M_{2}(\omega)=a^{-j \omega} M_{1}(\omega)$.

The magnitude of the Mellin transform of an input object or function is invariant to a scale change in the 
input, it is not invariant to a shift in the input function. Conversely, the magnitude of the Fourier transform of an object or function is invariant to a shift in the input function, but not to a scale change in the input. The scale and shift invariant transform required for correlation can be realized by forming the Mellin transform of the magnitude of the Fourier transform of the function [4].

Let us denote the two scaled versions of the input object or function as $f_{1}(x)=f(x)$ and $f_{2}(x)=f(a x)$, their Mellin transforms as $M_{1}$ and $M_{2}$, their Fourier transforms as $F_{1}$ and $F_{2}$ and the Mellin transforms of the magnitudes of $F_{1}$ and $F_{2}$ by $M_{1}^{\prime}$ and $M_{2}^{\prime}$. The Mellin-type correlation of two functions can be defined as

$f_{1}(x)(\Lambda) f_{2}(x)=\int_{0}^{\infty} f_{1}(y) f_{2}^{*}(x y)(1 / y) \mathrm{d} y$,

where $(\widehat{\Lambda}$ denotes the Mellin-type correlation. Substituting $x=\exp \xi$ and $y=\exp \eta$ into eq. (4), the conventional correlation of $f_{1}(\exp \xi)$ and $f_{2}(\exp \xi)$ results. The inverse Mellin transform can likewise be defined by

$M^{-1}\{M(\omega)\}=f(x)=(1 / 2 \pi) \int_{-\infty}^{\infty} M(\omega) x^{j \omega} \mathrm{d} \omega$.

Using these basic terms and the above notation, four possible methods of scale invariant correlation using Mellin transform can be distinguished.

(1) $M^{-1}\left\{M_{1} M_{2}^{*}\right\}=f_{1}(x)\left(\uparrow f_{2}(x)\right.$,

(2) $M^{-1}\left\{M_{1}^{\prime} M_{2}^{\prime *}\right\}=\left|F_{1}(\omega)\right|(\Lambda)\left|F_{2}(\omega)\right|$,

(3) $F\left\{M_{1} M_{2}^{*}\right\}=f_{1}(\exp x) \circledast f_{2}(\exp x)$,

(4) $F\left\{M_{1}^{\prime} M_{2}^{\prime *}\right\}=\left|F_{1}(\exp \omega)\right| \circledast\left|F_{2}(\exp \omega)\right|$.

Those correlation methods using the Fourier transform operation (Methods 3 and 4) rather than the inverse Mellin transform are clearly preferable for optical synthesis, since the Fourier transform is directly produced by a lens. Method 3 is not shift invariant since the functions rather than their Fourier transforms are operated on. Thus, implementations by this method would require precise placements of the input functions. Method 4 differs from Method 3 only in the additional initial step in which the magnitudes of the Fourier transforms $F_{1}$ and $F_{2}$ of the two functions $f_{1}$ and $f_{2}$ are formed. The basic operations in Method (3) are the same as those in Method (4), except for this initial step, and the resultant correlation in Method (3) is of the scaled space functions rather than the scaled transforms. Thus, Method (3) was chosen as the one to be implemented in these initial demonstrations of scale invariant correlation.

The optical implementation of a scale invariant correlation can be realized by the optical system of fig. 3. By $\log$ modules in the deflection system of whatever real-time input device is used $f_{1}(\exp \xi)$ and $f_{2}(\exp \xi)$ can be produced. With $f_{2}(\exp \xi)$ in plane $\mathrm{P}_{0}, M_{2}^{*}$ is formed and $\mathrm{P}_{1}$ by conventional holographic techniques [ 1 ], by interfering a plane wave off-axis reference beam and the Fourier transform of $f_{2}(\exp \xi)$. With the transmission of the input plane given by $f_{1}(\exp \xi)$, one component of the light distribution emerging from the transform plane can be described by $M_{1} M_{2}^{*}$. Its Fourier transform produced by $\mathrm{L}_{2}$ in plane $\mathrm{P}_{2}$ is proportional to $f_{1}(\exp \xi)$ $* f_{2}(\exp \xi)$ and is centered at $y=-f \sin \theta$ in this output plane (where $f$ is local length of the transform lenses used).

It can be shown that the $x$ displacement of the correlation peak in $\mathrm{P}_{2}$ is proportional to the scale factor $a$ between $f_{1}(x)=f(x)$ and $f_{2}(x)=f(a x)$. To implement the $x=\exp \xi$ scale conversion, the log of the coordinate of $f_{2}$ is formed. Denoting this coordinate as $x^{\prime}=a x$, we obtain

$\ln x^{\prime}=\ln a+\ln x$,

from which a scale in the input is seen to be equivalent to a shift by $\ln a$ in the new scaled functions. If $M_{1}(\omega)$ is the Mellin transform of the unscaled function, then

$M_{2}(\omega)=\exp (-\mathrm{j} \omega \ln a) M_{1}(\omega)$,

in agreement with eq. (3).

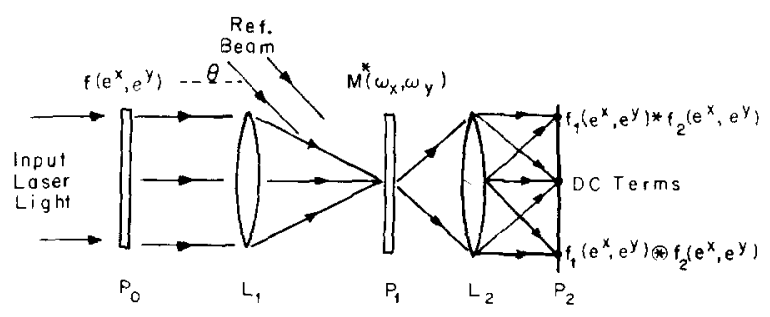

Fig. 3. Schematic of a scale invariant optical correlator using Mellin transforms. 


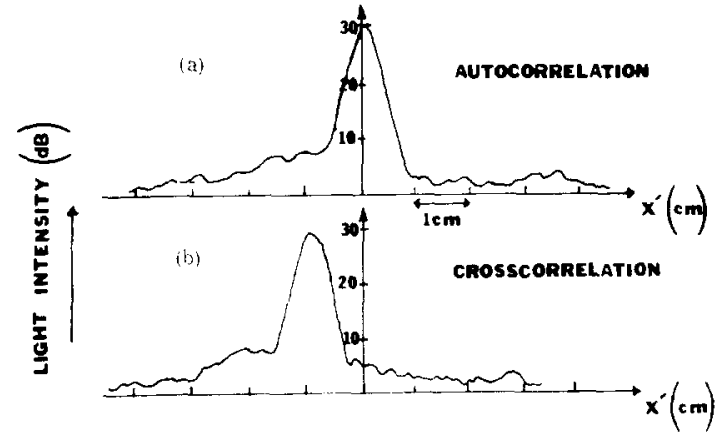

Fig. 4. Cross section of the optical correlation of two input squares that differ in scale by $200 \%$. (a) Auto correlation of the small square with a Mellin transform hologram of itself. (b) Correlation of the large square with the Mellin transform hologram of the small square.

Ignoring the effects of the reference beam angle $\theta$ on the $y$ coordinate of $M_{2}^{*}$ and considering only the one-dimensional function in the $x$ direction, the product $M_{1} M_{2}^{*}$ can be written as

$M_{1} M_{2}^{*}=M_{1}(\omega) M_{2}(\omega) \exp (-\mathrm{j} \omega \ln a)$.

The correlation, described by the Fourier transform of eq. (7) is then,

$f_{1}(\exp x) \circledast f_{2}(\exp x) \circledast \delta(x-\ln a)$

from which the $x$ coordinate of the correlation term $f_{1} \circledast f_{2}$ in $\mathrm{P}_{2}$ is seen to occur at $x=\ln a$. From the location of this correlation peak, the scale change $a$ between the input and filter function can thus be determined.

To demonstrate scale invariant correlation, transparencies of two simple square inputs which differ in scale by $200 \%$ were produced and the coordinates of these inputs were then logarithmically scaled. The scaled input for the smaller square was placed at $\mathrm{P}_{0}$ of fig. 3 and a Fourier transform hologram of it was formed on a $649 \mathrm{~F}$ spectrographic plate at $P_{1}$ with a $30^{\circ}$ reference beam angle $\theta$. The reference beam was then blocked and the cross-section through the autocorrelation peak in $\mathbf{P}_{2}$ was plotted using a scanning photometric microscope (see fig. $4 \mathrm{a}$ ). This procedure was repeated with the second larger input square placed in $\mathrm{P}_{0}$. The resultant cross-correlation is shown in fig. $4 \mathrm{~b}$. The SNRs of both correlation peaks are seen to be nearly identical. This confirms the scale invariance of this correlation and that objects that differ in scale can be correlated with no loss in the SNR of the correlation peak. The location of the correlation peak is shifted between correlations by $1 \mathrm{~cm}$ along the horizontal axis. This shift corresponds to $K \ln a=0.69 \mathrm{~K}$ $=1.035 \mathrm{~cm}$, where the constant multiplicative scale factor $K=1.5$ is a function of the synthesis and measurement methods used.

\section{Summary and conclusions}

A new approach to optical pattern recognition has been presented. The conventional Fourier transform operation inherent in an optical processor has been replaced by a Mellin transform. The resultant combined Fourier-Mellin transform has been shown to be invariant to a scale and shift in the input function. Four optical correlation methods using Mellin transforms have been presented, and evaluated. Experimental confirmation of the scale invariance of the basic Mellin transform-based optical correlator is presented.

These results confirm that it is possible to optically correlate two objects or functions that differ greatly in scale or size and that the scale and size differences in the two functions can be obtained from the position of the correlation peak. The importance of these and other new optical operations, which extend the flexibility of an optical processor, are of major importance in the implementation of a practical optical pattern recognition system.

\section{Acknowledgements}

This research was supported by the Office of Naval Research on Contract NR-350-011 and in part by the Air Force Office of Scientific Research, Air Force Systems Command USAF, under Grant No. AFOSR-752851. Initial technical discussions with Dr. George Huang of Philco-Ford are gratefully recognized.

The United States Government is authorized to reproduce and distribute reprints for governmental purposes notwithstanding any copywright notation hereon.

This manuscript is submitted for publication with the understanding that the United States Government is authorized to reproduce and distribute reprints for governmental purposes. 


\section{References}

[1] A. Vander Lugt, IEEE Trans. Info. Theory IT-10 (1964) 139.

[2] J.W. Goodman, Introduction to Fourier Optics (McGrawHill, New York, 1972).

[3] A. Vander Lugt, Proc. IEEE 62 (1974) 1300.

[4] D. Casasent and D. Psaltis, Opt. Engr. (Feb. 1976) accepted for publication.
[5] G. Huang, F. Russell and W. Chen, EIA Symp. (College Park, Apr. 1975).

[6] R. Bracewell, The Fourier Transform and Its Applications, (McGraw-Hill, New York, 1963) Chap. 12.

[7] F.R. Gerardi, IRE Trans. Circ. Theory (June 1959) 197-208.

[8] A. Sawchuk, J. Opt. Soc. Amer. 64 (1974) 138.

[9] S. Cohn-Sfetchu, M. Smith and S. Nichols, Proc. IEEE (Lett.) (Feb. 1975) 326. 